\title{
Citrus aurantium Blossom and Preoperative Anxiety
}

\author{
Mahmood Akhlaghi ${ }^{1}$, Gholamreza Shabanian ${ }^{2}$, Mahmoud Rafieian-Kopaei ${ }^{3}$, Neda Parvin ${ }^{4}$, Mitra Saadat ${ }^{5}$, \\ Mohsen Akhlaghi 6
}

\begin{abstract}
Summary: Akhlaghi M, Shabanian G, Rafieian-Kopaei M, Parvin N, Saadat M, Akhlaghi M - Citrus aurantium Blossom and Preoperative Anxiety.

Background and objectives: Reducing anxiety is very important before operation. Preoperative visit and use of premedication are popular methods to achieve this goal, but the role of anxiolytic premedication remains unclear and postoperative side-effects may result from routine premedication. Citrus aurantium is used as an alternative medicine in some countries to treat anxiety, and recently the anxiolytic role of this medicinal plant was established in an animal model study. The aim of this study was to assess the anxiolytic effect of Citrus aurantium blossom on preoperative anxiety.
\end{abstract}

Methods: We studied 60 ASA I patients undergoing minor operation. In a randomized double-blind design, two groups of 30 patients received one of the following oral premedication two hours before induction of anesthesia: 1) Citrus aurantium blossom distillate $1 \mathrm{~mL} . \mathrm{kg}^{-1}$ (C-group); 2) Saline solution $1 \mathrm{~mL} . \mathrm{kg}^{-1}$ as placebo (P-group). Anxiety was measured before and after premedication using the Spielberger state-trait anxiety inventory (STAl-state) and the Amsterdam preoperative anxiety and information scale (APAIS) before operation.

Results: After premedication, both the STAl-state and the APAIS scales were decreased in C-group $(p<0.05)$; while exhibiting no significant changes in P-group.

Conclusions: Citrus aurantium blossom may be effective in terms of reduction in preoperative anxiety before minor operation.

Keywords: Citrus; Flowers; Preoperative Care; Anxiety; Ambulatory Surgical Procedures.

@2011 Elsevier Editora Ltda. All rights reserved.

\section{INTRODUCTION}

There is no doubt that people awaiting surgery experience anxiety ${ }^{1}$ This phenomenon is closely related to fear from a new unfamiliar environment, fear of surgery and death ${ }^{2}$. Incidences of preoperative anxiety have been reported from $11 \%$ to $80 \%$ of adult patients. Related to this large number of preoperative anxious patients, preoperative anxiety could influence on the course and outcomes of surgical treatments ${ }^{3}$.

Received from Medicinal Plants Research Center, (Shahrekord University of Medical Sciences), Shahrekord, Iran.

1. MD; Associate Professor of Anesthesiology, Anesthesiology Department, Shahrekord University of Medical Sciences, Shahrekord, Iran

2. MD; Assistant Professor of Anesthesiology, Medicinal Plants Research Center, Shahrekord University of Medical Sciences, Shahrekord, Iran

3. PhD; Professor of Pharmacology, Medicinal Plants Research Center, Shahrekord University of Medical Sciences, Shahrekord, Iran

4. MA; Lecturer of Nursing, Medicinal Plants Research Center, Shahrekord University of

Medical Sciences, Shahrekord, Iran

5. MD; General Practitioner, Medicinal Plants Research Center, Shahrekord University of Medical Sciences, Shahrekord, Iran

6. Pharmacy Student, Shiraz University of Medical Sciences, International Branch, Shiraz, Iran

Submitted on March 4, 2011.

Approved on March 28, 2011.

Correspondence to:

Dr. Gholamreza Shabanian

Medicinal Plants Research Center

Shahrekord University of Medical Sciences,

8815713471 - Shahrekord, Iran

E-mail: akhlaghi236@yahoo.com
The correlation between preoperative anxiety and some complications such as postoperative pain, postoperative intravenous sedation, or more anesthetic requirements have been established by some studies ${ }^{4-7}$. Maranets et al. ${ }^{5}$ found that high baseline anxiety level predicts increased intraoperative anesthetic requirements and they suggest that anesthesiologists should modify the initial induction dose based on the anxiety level exhibited by patients.

Reducing anxiety is very important before operation. Several methods have been suggested towards reducing or controlling this psychological problem related to operation. Pre-anesthesia assessment in an outpatient clinic as well as visit during hospitalization in the evening just before surgery may reduce preoperative anxiety ${ }^{8}$.

Besides the advantages of preoperative visit and reassurance of the patient before surgery, premedication with anxiolytic and sedative drugs may reduce preoperative anxiety ${ }^{9}$. On the other hand, the role of anxiolytic premedication remains unclear and postoperative side-effects may result from routine premedication ${ }^{10}$.

Citrus aurantium, commonly known as sour orange or bitter orange (local name in Iran: Nareng) is produced in Northern and Southern Iran. Traditionally, Citrus aurantium is used as an alternative medicine in some countries to treat anxiety, insomnia and as an anticonvulsant, suggesting depressive action upon the central nervous system (CNS) ${ }^{11}$. In an anxiety model study, Citrus aurantium was able to enhance the sleeping time induced by barbiturates. In animal model, this sedative effect was in accordance with traditional use of citrus aurantium ${ }^{11}$. 
In spite of traditional use of Citrus aurantium for reducing anxiety and its sedative effect in animal model ${ }^{12}$, we believe that preoperative anxiolytic effect of Citrus aurantium blossom has not been studied so far. The present study was designed to assess the effect of Citrus aurantium blossom on preoperative anxiety in patients scheduled for elective minor surgery.

\section{METHODS}

Ethical approval for this study (Ethical Committee No. 87-8-4) was provided by the Ethical Committee of Shahrekord University of Medical Sciences, Shahrekord, Iran (Prof. H. Yousefi) on February 9, 2009. Written informed consent was obtained from 60 consecutive outpatients, aged 15-60 year, ASA physical status I scheduled for lower limb minor operation under general anesthesia. Preoperative visit was done by an anesthetist the day before operation. Exclusion criteria were coexisting CNS diseases, malignancy, a variety of neuropsychological disorders, use of medications including drugs related to surgery, and any history of smoking or opium addiction, as well as any cardiovascular disease. None of the patients had previous anesthesia and surgical experiences as well as any history of hospitalization. Routine sedative premedication was not offered to patients participating in this study, instead premedication with either Citrus aurantium blossom distillate (CABd) or placebo was assumed for two groups of study two hours before operation. Patients enrolled in this study were assigned into two groups of 30 each; using a computer-base random allocation and double blind manner.

Fresh petals and stamens of Citrus aurantium blossoms were collected from adult sour orange plants, existing in south Iran, at Medicinal Plants Research Center of Shahrekord University of Medical Sciences. Citrus aurantium blossom distillate (CABd) was obtained by steam distillation and then protected until pharmacological assays. CABd was standardized based on measurement of linalool, total phenolic and flavonoid compounds as follows:

Linalool concentration was determined by a reversed phase HPLC method (C18 column, Agilent, Germany) ${ }^{13}$. An ultraviolet (UV) detector was used with wavelength of $254 \mathrm{~nm}$ to obtain the chromatograph corresponding to linalool. Mobile phase consisted of $0.05 \mathrm{M}$ phosphate buffer ( $\mathrm{pH}$ adjusted to 3 ) and acetonitrile at a rate of 50:50. Flow rate was $1.3 \mathrm{~mL} \cdot \mathrm{min}^{-1}$ and injection volume was $100 \mu \mathrm{L}$. A standard curve was drawn using the area under the curves resulted from different doses of linalool. The experiment was repeated for three times and the amount of linalool in the sample was determined using this standard curve.

The amount of total phenolic compounds in CABd was determined colorimetrically with the Folin-Ciocalteu reagent, using the method described by $\mathrm{Kim}^{14}$. A solution of $5 \mathrm{~mL} \mathrm{CABd}$ or Gallic acid (standard phenolic compound) was mixed with Folin Ciocalteu reagent (1:10 diluted with distilled water) and aqueous $\mathrm{Na}_{2} \mathrm{CO}_{3}(4 \mathrm{~mL}, 1 \mathrm{M})$. The mixtures were allowed to stand for $15 \mathrm{~min}$ and the total phenols were determined by colorimetry at $765 \mathrm{~nm}$. A standard curve was prepared using
$0,50,100,150,200$, and $250 \mathrm{mg} . \mathrm{L}^{-1}$ solutions of Gallic acid in methanol: water $(50: 50, \mathrm{v} / \mathrm{v})$. Total phenol values were expressed in terms of Gallic acid equivalent $\left(\mathrm{mg}^{-1} \mathrm{~g}^{-1}\right)$, which is a common reference compound. The experiment was repeated for three times.

The amount of total flavonoids in the $C A B d$ was determined using colorimetric method as described by Chang ${ }^{15}$. A solution of $0.5 \mathrm{~mL}$ of CABd or Rutin (standard flavonoid compound) was mixed with $1.5 \mathrm{~mL}$ of methanol, $0.1 \mathrm{~mL}$ of $10 \%$ aluminum chloride, $0.1 \mathrm{~mL}$ of $1 \mathrm{M}$ potassium acetate and $2.8 \mathrm{~mL}$ of distilled water and remained at room temperature for $30 \mathrm{~min}$. Then the absorbance of the reaction mixture was measured at $415 \mathrm{~nm}$ with a double beam spectrophotometer (Unico UV2100 , Japan). The calibration curve was prepared using Rutin solutions at concentrations of 25 to $500 \mathrm{ppm}$ in methanol. The experiment was repeated for three times. Total flavonoids were expressed in terms of Rutin equivalent $\left(\mathrm{mg}^{-\mathrm{g}^{-1}}\right)$, which is a common reference compound.

The retention time of linalool with the condition described in Methods section was $2.8 \mathrm{~min}$. The amount of linalool in the sample was $10 \mathrm{ppm}$ (part per million). Total phenolic contents of CABd were $33 \pm 0.4 \mathrm{mg} \cdot \mathrm{g}^{-1}$ equivalent to Gallic acid and

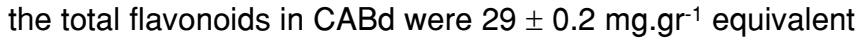
to Rutin.

The average amounts of total flavonoid and phenolic compounds were $29 \pm 0.2$ (mg.g ${ }^{-1}$ Rutin) and $33 \pm 0.4$ (mg.gr-1 Gallic acid), respectively. The linalool concentration in the sample was $10 \mathrm{ppm}$. Finally, the concentrations of total flavonoid, phenolic compound and linalool in CABd were $29 \mathrm{mg} \cdot \mathrm{mL}^{-1}$, $33 \mathrm{mg} \cdot \mathrm{mL}^{-1}$ and $0.01 \mathrm{mg} \cdot \mathrm{mL}^{-1}$, respectively.

Preoperatively, all patients were admitted to the same holding area near the operating room while waiting for outpatient surgery. For all subjects the environment, the nursing staff and the anesthesiologist as well as the psychologist were the same in the holding area. As a baseline, preoperative anxiety was assessed using both State-Trait Anxiety Inventory (STAIstate) and Amsterdam Preoperative Anxiety and Information Scale (APAIS) by an expert psychologist. Heart rate (HR) and blood pressure (BP) were also obtained two hours before operation just before premedication in the holding area by a nurse anesthetist. Then subjects of the two groups received oral CABd $1 \mathrm{~mL} . \mathrm{kg}^{-1}$ as test (C - group) or Saline solution $1 \mathrm{~mL} . \mathrm{kg}^{-1}$ as placebo ( $\mathrm{P}$ - group), respectively; according to a computer based randomization just after collecting the baseline values. Two hours later right before inducing anesthesia anxiety was assessed using the same methods by other anesthesiologist and psychologist who were blinded to the groups and patients' baseline values.

The State-Trait Anxiety Inventory (STAl) is a 40 -item self report measure that contains 20 items measuring state anxiety and 20 items measuring trait anxiety ${ }^{2}$. Total scores for state and trait sections separately range from 20 to 80 with higher scores denoting higher levels of anxiety. Amsterdam Preoperative Anxiety and Information Scale (APAIS) consist of 6 questions and ranged from 6-30. The APAIS was further subdivided into subscales in order to separate anxiety about anesthesia (sum of anesthesia anxiety "sum A" questions 
one and two), anxiety regarding surgery (sum of surgery anxiety "sum S" questions four and five) and a total of two scores (sum of combined anesthesia and surgery anxiety "sum $C=$ sum $A+$ sum $S$ ") ${ }^{16}$. In this study, we used state component of STAl (rage 20-80) and sum C component of APAIS (range 4-20) to evaluate preoperative anxiety.

Both heart rate $(\mathrm{HR})$ and blood pressure $(\mathrm{BP})$, which have been widely used as dependent variables in behavioral studies to alter levels of anxiety and are frequently cited as physiological indices of stress in psychology, aviation medicine, and anesthesia ${ }^{2}$ were obtained in two steps as baseline and just before induction of general anesthesia.

Regarding the normal distribution of variables, the data between groups were analyzed using Chi-Square and independent $t$ tests and the data within groups were analyzed using paired $t$ test. In all tests, a $\mathrm{p}$-value $<0.05$ was considered to indicate statistical significance.

\section{RESULTS}

Sixty patients were enrolled and completed the study. ChiSquare test demonstrated no significant difference among male and female distribution between groups. According to independent $t$ test no significant differences were seen between groups in terms of age, baseline values of STAI-state, APAIS, and baseline hemodynamic values. (Table I)

Paired Student's $t$ test showed that patients in C-group were significantly less anxious than patients in P-group when both STAI-state and APAIS were considered for anxiety evaluation $(p<0.05)$. Hemodynamic variables (except pulse rate in group $P$ ), were not changed within the two groups after premedication when paired $t$ test was used. (Tables II and III)

\section{DISCUSSION}

Preoperative anxiety is not only an unpleasant emotional state but may lead to significant psychological and physiological disorders ${ }^{16}$. The main finding of this study is that the anxiolytic effect of Citrus aurantium blossom distillate (CABd) when used orally was highly significant as measured by STAI-state and APAIS. Both STAI-state and APAIS were decreased by CABd. On the other hand, neither STAI-state nor APAIS was changed in the placebo group.

We found no studies related to the effect of CABd on preoperative anxious situation or even anxiolytic effect of this herbal medicine in human in the literature review. Only a few studies

Table I - Data of Baseline STAI-State, APAIS, and Hemodynamic Values

\begin{tabular}{|c|c|c|c|c|c|}
\hline Variables & $\begin{array}{l}\text { C-group } \\
(\text { Mean } \pm \text { SD) }\end{array}$ & $\begin{array}{l}\text { P-group } \\
(\text { Mean } \pm \text { SD) }\end{array}$ & \multicolumn{2}{|c|}{$95 \%$ Confidence Interval } & $\mathrm{p}$-value \\
\hline STAl-state* & $52.23 \pm 10.35$ & $52.60 \pm 9.79$ & -5.57 & 4.84 & 0.888 \\
\hline APAIS* & $10.90 \pm 2.09$ & $10.80 \pm 2.36$ & -1.05 & 1.25 & 0.863 \\
\hline Pulse Rate* (bpm) & $79.46 \pm 12.29$ & $81.80 \pm 13.65$ & -9.04 & 4.38 & 0.489 \\
\hline Systolic BP* $(\mathrm{mmHg})$ & $122.00 \pm 8.76$ & $125.33 \pm 13.46$ & -9.20 & 2.53 & 0.260 \\
\hline Diastolic BP* (mmHg) & $85.33 \pm 7.91$ & $84.50 \pm 10.55$ & -3.98 & 5.65 & 0.731 \\
\hline
\end{tabular}

STAI-state: state anxiety score of the standard Spielberger state-trait anxiety inventory; APAIS: Amsterdam preoperative anxiety inventory scale; ${ }^{\star}$ No significant differences were seen between groups using independent $t$ test.

Table II - Differences of STAI-State, APAIS, and Hemodynamic Values before and after Premedication in C-group

\begin{tabular}{|c|c|c|c|c|c|}
\hline Variables & $\begin{array}{l}\text { Differences } \\
\text { (Mean } \pm \text { SD) }\end{array}$ & $\begin{array}{l}\text { Standard Error } \\
\text { (Mean) }\end{array}$ & Lower & Upper & $\mathrm{p}$-value \\
\hline APAIS* & $1.80 \pm 0.80$ & 0.14 & 1.49 & 2.10 & 0.000 \\
\hline Systolic BP (mmHg) & $1.33 \pm 8.50$ & 1.55 & -1.84 & 4.50 & 0.397 \\
\hline Diastolic BP (mmHg) & $0.10 \pm 1.86$ & 0.34 & -0.59 & 0.79 & 0.771 \\
\hline
\end{tabular}

STAI-state: state anxiety score of the standard Spielberger state-trait anxiety inventory; APAIS: Amsterdam preoperative anxiety inventory scale; ${ }^{\star}$ Significant difference using paired $t$ test $(\mathrm{p}<0.05)$. 
Table III - Differences of STAI-State, APAIS, and Hemodynamic Values before and after Premedication in P-group

\begin{tabular}{|c|c|c|c|c|c|}
\hline Variables & $\begin{array}{l}\text { Differences } \\
\text { (Mean } \pm \text { SD) }\end{array}$ & $\begin{array}{l}\text { Standard Error } \\
\text { (Mean) }\end{array}$ & \multicolumn{2}{|c|}{ 95\% Confidence Interval } & $p$-value \\
\hline APAIS & $0.26 \pm 1.11$ & 0.20 & -0.14 & 0.68 & 0.199 \\
\hline Systolic BP (mmHg) & $-1.86 \pm 7.80$ & 1.42 & -4.78 & 1.04 & 0.201 \\
\hline Diastolic BP (mmHg) & $0.26 \pm 6.24$ & 1.14 & -2.06 & 2.59 & 0.817 \\
\hline
\end{tabular}

STAI-state: state anxiety score of the standard Spielberger state-trait anxiety inventory; APAIS: Amsterdam preoperative anxiety inventory scale; ${ }^{*}$ Significant difference using paired $t$ test $(p<0.05)$.

reported that some similar components of citrus may reduce anxiety ${ }^{17,18}$. Lehrner et al. 17 found that women exposed to orange odor before dental surgery had a lower level of state anxiety, a more positive mood and a higher level of calmness. Although the main components of the essential oil in their study, demonstrated by gas chromatography, was in part different from that obtained in our study, the essential similar component was limonene, a flavonoid compound, which could be addressed as anxiolytic component.

The main component of CABd in our study was not similar to that of Carvalho and Costa ${ }^{11}$. They obtained d-limonene $90.4 \%$ extracted from peel and leaves of citrus. In their study, sleeping time induced by pentobarbital, anxiolytic activity, and anticonvulsant activity were evaluated in mice model. The result of their study suggested sedative/hypnotic activity of Citrus aurantium.

Chemical analysis of flowers of Sour orange has been previously reported by an investigation as flavonoids, which are its main constituents ${ }^{19}$. Flavonoids are complex chemical molecules that may act as ligands for benzodiazepine receptors ${ }^{20}$. The notion that flavonoids may be agonists of benzodiazepine receptors, suggest that the flavonoid, which was extracted from the CABd in our study, might behave as an agonist of benzodiazepine receptors and reduce preoperative anxiety.

The phenolic component threshold toxicity level has been confirmed between 1050 and $1600 \mathrm{mg} \cdot \mathrm{L}^{-1}$, as stated in other studies, the concentration components of CABd used in our study was too low to induce toxicity in the patients ${ }^{21-24}$. However, based on traditional use of this herb in our society, we believe that the dose of $C A B d$ used in this study has no side effect on physiological responses. Fortunately, we observed no adverse effect during and after operation.

Citrus aurantium interferes with many drugs. Some of these drugs related to anesthesia are anti-anxiety, anti-hypertensive agents, and sedatives as well as antiemetic agents $25-27$. Although these interactions are due to Citrus aurantium peel and fruit and there is no study establishing the interaction of CABd components with other medications, the patients with no history of medication and disease were included to prevent any bias in the study.

Despite anxiety related to anesthesia and surgery, there are several sources of fear that may interfere with an anxiety model study. The main preoperative anxiety risk factors have been listed in recent studies. These studies concluded that concern history about cancer, psychiatric disorders, depression, trait-anxirty level, postoperative pain, history of smoking, extend of the proposed surgery, and physical status according to ASA, as well as patient education are the main risk factors for anxious situation before surgery ${ }^{3,28-30}$. We circumvented some problems using the same situation, such as holding area for all patients, choosing the patients scheduled for minor lower limb surgery, sharing the same operating room staff and preventing needling and any stress induced factors before evaluating anxiety and excluding subjects with some mentioned risk factors. Moreover, all participants had no history of using herbal medicine or any other medication on questioning.

We used both the Spielberger state-trait anxiety inventory (STAI) scale and the Amsterdam preoperative anxiety and information scale (APAIS) to evaluate preoperative anxiety. Boker's study confirmed significant and positive correlation between sum C (sum of "Anesthesia anxiety" and "Surgery anxiety") of APAIS and STAl-state scales ${ }^{16}$. The result of our study completely agrees with Boker's study.

Several advantages related to our study have to be addressed. First, the study is a double-blind randomized trial that enrolled appropriate number of patients with ASA I scheduled for minor outpatient surgery without any experience of previous hospitalization and surgery. Second, the use of both STAI-state and APAIS could reduce any bias while evaluating preoperative anxious situation. Third, regarding preoperative factors that may interfere with the study, we tried to omit some factors by choosing similar proper place and the same staff in the study. 
Unfortunately, some problems related to this study are as follow: We were not able to record electrodermal activity (EDA) as a measure of autonomic arousal in response to stress. We also did not determine plasma cortisol or plasma catecholamine ${ }^{2}$, in our study. These problems were circumvented using two methods of anxiety evaluation.
In conclusion, our study demonstrated that CABd may reduce preoperative anxiety in outpatient surgery. This result could open a window towards introducing herbal medicine for premedication as well as educate the anesthesiologist about using herbs and possible interaction with routine premedication. 